Scientific article

Los textos publicados son responsabilidad exclusiva de sus autores

\title{
Relación entre rendimiento académico y estilos de aprendizaje
}

\section{Relationship between academic performance and learning styles}

\section{Referencia del artículo}

\author{
- Ingrid Marisol Yumán Ramírez \\ - Maestría en Docencia Universitaria \\ Facultad de Humanidades de Universidad de San \\ Carlos de Guatemala \\ yumanramirezingrid@gmail.com \\ https://orcid.org/0000-0002-3024-8790
}

Yumán-Ramírez, I. M. (2020). Relación entre rendimiento académico y estilos de aprendizaje. Revista Guatemalteca de Educación Superior, 3(2), 1-11.

DOI: https://doi.org/10.46954/revistages.v3i2.27

$\begin{array}{ll}\text { Recibido } & 07 / 07 / 2020 \\ \text { Revisado } & 07 / 08 / 2020 \\ \text { Aceptado } & \text { 23/08/2020 }\end{array}$

\section{Resumen}

En el año 2000 la Universidad de San Carlos de Guatemala implemento el Programa Académico Preparatorio -PAPconstituyéndose en un programa permanente, continuo y sistemático, cuyo propósito es nivelar los conocimientos de ciencias básicas de los aspirantes a ingresar a la USAC, que obtienen un resultado insatisfactorio en las Pruebas Básicas. Cada año se inscriben al PAP 1200 estudiantes, de los cuales solo el $25 \%$ logra obtener resultado satisfactorio, dejando la inquietud de conocer cuáles son los estilos de aprendizaje de los estudiantes del PAP y sus efectos en el rendimiento académico. El objetivo de la investigación es identificar los estilos de aprendizaje y su relación con el rendimiento académico, la población fue de 418 estudiantes, de 8 secciones entre las edades de 16 a 32 años, de los cuales 104 fueron mujeres y 314 hombres, para conocer los estilos de aprendizaje se aplicó una escala de Likert, con valores de acuerdo y desacuerdo en escala de 1 a 5, dando como resultado que 301 alumnos 
Facultad de Humanidades

Escuela de Estudios de Postgrado

Palabras clave: estilos de aprendizaje, programa académico preparatorio, rendimiento académico.

Keywords: learning styles, academic preparatory program, academic achievement tiene un aprendizaje visual, 78 un aprendizaje auditivo y 39 un aprendizaje kinestésico. Predominando el aprendizaje visual con el $72 \%$ de la población total. Para identificar la relación entre estilos de aprendizaje y rendimiento académico se realizó un análisis correlación lineal, entre la nota obtenida en el curso de matemática y el valor promedio (de 1 a 5) obtenido en la escala de Likert, dando como resultado que ninguno de los estilos de aprendizaje está relacionado con el rendimiento académico.

\section{Abstract}

In 2000, the University of San Carlos de Guatemala implemented the Academic Preparatory Program -PAP-, becoming a permanent, continuous and systematic program, the purpose of which is to level the basic science knowledge of applicants to enter the USAC, who obtain a unsatisfactory result in the Basic Tests. Every year 1,200 students enroll in the PAP, of which only $25 \%$ manage to obtain satisfactory results, leaving the concern of learning about the learning styles of the PAP students and their effects on academic performance. The objective of the research is to identify learning styles and their relationship with academic performance, the population was 418 students from sections $A$ to $\mathrm{H}$, between the ages of 16 to 32 years, of whom 104 were women and 314 Men, to know the learning styles, a Likert scale was applied, with values of agreement and disagreement on a scale of 1 to 5 , resulting in 301 students having visual learning, 78 hearing learning and 39 kinesthetic learning. Prevailing visual learning with $72 \%$ of the total population. To identify the relationship between learning styles and academic performance, a linear correlation analysis was performed, between the grade obtained in the mathematics course and the average value (from 1 to 5) obtained on the Likert scale, resulting in that none of learning styles is related to academic performance.

\section{Introducción}

El presente estudio se realizó considerando la importancia de dar respuesta a las posibles deficiencias que pueden presentar los estudiantes del Programa Académico Preparatorio de la Facultad de Ingeniería, en cuanto a los estilos de aprendizaje 
y su repercusión en el rendimiento académico, ya que se ha observado que un alto porcentaje de estudiantes no logran alcanzar las competencias y las destrezas esperadas, obteniendo resultado insatisfactorio, presentándose casos de hasta tres años de repitencia y de constantes fracasos al someterse a las pruebas de Conocimientos Básicos, lo que ha repercutido en la salud mental del estudiante, presentando problemas de baja autoestima, ansiedad, inseguridad, desinterés por aprender y en la esperanza de una vida mejor.

La importancia de la investigación radica en dar respuesta a las dificultades del aprendizaje que se han observado y en fortalecer las debilidades en la formación y adquisición de conocimiento, por lo que se propuso identificar los estilos de aprendizajes y su relación en el rendimiento académico de los estudiantes del PAP de Ingeniería de la USAC. y con esta base proponer estrategias de aprendizaje que beneficien el rendimiento académico de los alumnos y lograr el ingreso y permanencia en la USAC. Para iniciar este estudio empezaremos por determinar las variables que se trabajaron:

\section{Variable 1: Estilos de Aprendizaje}

El concepto de Estilos de Aprendizaje de acuerdo con (Alonzo, 2007) define varios conceptos: Hunt (1979, como cito en Alonzo, 2007) describe estilos de aprendizaje como "las condiciones educativas bajo las que una persona está en la mejor situación para aprender, o qué estructura necesita la persona para aprender mejor". P.79. Los Estilos de Aprendizaje son los rasgos cognitivos, afectivos y fisiológicos, que sirven como indicadores relativamente estables, de cómo los estudiantes perciben, interaccionan y responden a sus ambientes de aprendizaje. Cuando hablamos de estilos de aprendizaje estamos teniendo en cuenta los rasgos cognitivos, incluimos los estudios de psicología cognitiva que explica la diferencia en los sujetos respecto a la forma de conocer. Este aspecto cognitivo es el que caracteriza y se expresa en los estilos Cognitivos.

Otra definición de Estilos de Aprendizaje es la de (Woolfolk, 2010) "Un estilo de aprendizaje se define como los métodos que 
una persona utiliza para aprender y estudiar". P.121 Resaltando la importancia que se debe de promover en los estudiantes la autoconciencia y la autoevaluación de sus propios estilos de aprendizaje para estudiar con motivación desde las herramientas que les faciliten la adquisición del aprendizaje, lo cual contribuirá a que el docente observe sus diferentes técnicas y las adapte al proceso de enseñanza.

(Grinder \& y Bandler, 2004) desarrollaron la teoría de los estilos de aprendizaje Visual, Auditivo y Kinestésico también llamado VAK por sus siglas, esta teoría plantea que las personas responden a los estímulos sensoriales los cuales entran por los sentidos visual, auditivo y kinestésico, la información es captada a través del sentido que la persona tiene más desarrollando y almacenada en la memoria como un mapa conceptual y relacionada con la experiencia previa de cada individuo luego es traída al presente si necesitamos recordar algo.

Esta propuesta teórica fue la base de la investigación sobre los estilos de aprendizaje ya que presenta la información mediante diferentes enfoques, llevándonos a una instrucción más efectiva y aporta ventajas que pueden justificar la interiorización del conocimiento, la posibilidad de utilizar otros métodos en la práctica educativa, la fácil actualización de los contenidos, la existencia de un feed-back de información inmediato, la motivación y mejora en el proceso de aprendizaje del alumno; de manera que el profesor conoce si el alumno responde al método de enseñanza y alcanza los objetivos fijados inicialmente. Conozcamos un poco más de cada uno de los estilos de aprendizaje propuestos por Grinder y Bandler:

En el aprendizaje visual el alumno aprende a través del canal visual (viendo). Le gusta obtener la mayor estimulación visual posible, prefiere la lectura y el estudio de gráficas, a este tipo de estudiante las conferencias, conversaciones e instrucciones orales sin un apoyo visual le pueden producir ansiedad y resultar confusas. Reid (1995) agrega que estos estudiantes requieren del estímulo visual de tableros informativos, videos, películas, palabras escritas en el pizarrón, un libro o libreta de notas, ya que recordarán y comprenderán mejor la información e instrucciones que reciban 
a través del canal visual. Si asisten a una conferencia o reciben instrucciones en forma verbal, les conviene tomar notas.

En el aprendizaje Auditivo Según (Reid, 1995) el estudiante a través del oído (escuchando). Es del tipo de estudiante que aprende más a través de explicaciones orales. Puede recordar y comprender mejor la información si lee en voz alta o si mueve los labios mientras lee, especialmente cuando se trata de material nuevo. Puede beneficiarse al escuchar cintas electromagnéticas, conferencias, discusiones en clase, enseñando a otros compañeros o bien conversando con el profesor.

Y en el aprendizaje Kinestésico (Reid, 1995) manifiesta "que este tipo de estudiante aprende mejor a través de la experiencia" P. 3 Saca mayor provecho al involucrarse en actividades físicas en el aula. Su participación activa en las diferentes tareas, viajes y juegos de roles en el salón de clase le ayudarán a recordar mejor la información. A este tipo de estudiante estar sentado en un escritorio por muchas horas le resulta incómodo, necesita descansos frecuentes y, sobre todo, acción física en juegos y actividades dramáticas.

\section{Variable 2. Rendimento Académico}

El rendimiento académico hace referencia a la evaluación del conocimiento adquirido en el ámbito escolar, terciario o universitario. El rendimiento académico es una medida de las capacidades del alumno que expresa lo que está el aprendiendo a lo largo del proceso formativo. El rendimiento Académico también se puede ver afectado por factores externos al estudiante en los ámbitos familiares, económicos o culturales. Según (Figueroa, 2009) "El rendimiento académico se define como el producto de la asimilación del contenido de los programas de estudio" p.33, en otras palabras el rendimiento académico, es el resultado cuantitativo que se alcanza de la asimilación y evaluación de los aprendizajes adquiridos, es un indicador del nivel o grado de conocimientos, destrezas, competencias y habilidades alcanzado por el estudiante en el proceso de evaluación a través de la nota numérica establecida para su aprobación según el reglamento de evaluación. 


\section{Materiales y Métodos}

El diseño de la investigación presentada es el descriptivo no experimental, correlacionado con dos variables los estilos de aprendizaje y el rendimiento académico, la fuente de investigación fueron alumnos del programa académico preparatorio y el registro de notas obtenidas por los estudiantes durante el curso de matemáticas que se desarrolló en el primer semestre del año 2020.

La población con la que se trabajó fue de 1200 estudiantes, inscritos en el Programa Académico Preparatorio de la Facultad de Ingeniera en las tres jornadas, plan diario, en el primer semestre del 2020, con un nivel académico de diversificado. La muestra total fue de 418 estudiantes con un muestreo no probabilístico. El alcance de la investigación fue correlacional lineal, identificando los estilos de aprendizaje de los estudiantes del Programa Académico Preparatorio y su relación con el rendimiento académico.

Para tener acceso a la población en la modalidad virtual se solicitó el apoyo de la coordinación del Programa Académico Preparatorio, utilizando la escala de Likert en un formulario de google forms, el cual fue aplicado a los estudiantes en un tiempo aproximado de siete a diez minutos, considerando los criterios de inclusión como estar legalmente inscrito en el PAP, ser estudiante regular y aceptar el consentimiento informado por parte del estudiante para ser parte del estudio.

La información que se capturó fue analizada y comparada con la base de datos de notas de los estudiantes del PAP, la cual fue proporcionada por la coordinación del Programa Académico Preparatorio, bajo confiabilidad y para usos exclusivos de la presente investigación.

\section{Resultados}

La muestra que se trabajó está conformada por estudiantes del nivel medio, entre las edades de 16 a 32 años, de los cuales 104 son mujeres y 314 son hombres quienes están inscritos en el Programa de Académico Preparatorio, durante el primer semestre del año 
2020. De los cuales se pudo determinar lo siguiente con respecto a su estilo de aprendizaje.

Objetivo específico 1: Identificar los estilos de aprendizaje de los estudiantes del PAP.

De acuerdo con la definición de las variables y unidades de análisis para la variable aprendizaje se utilizó la escala de Likert para obtener valores de acuerdo y desacuerdo en escala de 1 a 5 , de la pregunta 1 a la pregunta 8 se evaluó el estilo de aprendizaje visual, de la pregunta 9 a la pregunta 13 se evaluó el estilo de aprendizaje auditivo y de la pregunta 14 a la pregunta 20 se evaluó el estilo kinestésico; para determinar el estilo que define a un estudiante se realizó un promedio ponderado de los valores obtenidos del grupo de preguntas de cada estilo, el estilo con mayor valor en el promedio representó el estilo de aprendizaje que representa a cada uno de los estudiantes. En la tabla 1 se muestra el resultado por estilo de aprendizaje de un total de 418 estudiantes entrevistados.

\section{Tabla N 1}

Cantidad de estudiantes clasificados por el estilo de aprendizaje, Programa Académico Preparatorio (PAP) jornada matutina, abril 2020.

\begin{tabular}{ccc}
\hline Estilos de aprendizaje & Total & Porcentaje \\
\hline Auditivo & 78 & 18.66 \\
Kinestésico & 39 & 9.33 \\
Visual & 301 & 72.01 \\
\hline Total & 418 & 100 \\
\hline
\end{tabular}

Fuente: elaboración propia, con resultados de la investigación.

Con los datos representados en la tabla 1 se realizó el cálculo del estadístico chi cuadrado de Pearson (286.83) dando como resultado un valor probabilidad ( $p$-valor) menor a 0.0001 , lo cual evidencia claramente que los estudiantes del Programa Académico Preparatorio (PAP) jornada matutina tienen diferentes estilos de aprendizaje, dentro de los cuales predomina el estilo visual con $72 \%$ del total de la muestra de estudiantes. 
Al analizar la relación que existe entre los estilos de aprendizaje y su efecto en el rendimiento académico de los estudiantes de Programa Académico Preparatorio (PAP) se realizó un análisis de correlación entre la nota obtenida y el valor promedio (de 1 a 5) obtenido por el estudiante para el estilo de aprendizaje sobresaliente para cada uno. Este valor promedio del estilo de aprendizaje sobresaliente por estudiante fue obteniendo del valor medio del resultado de cada una de las preguntas que conforman cada estilo de aprendizaje, los cuales se encuentran descritos en la tabla de definición de las variables y unidades de análisis.

\section{Tabla N 2}

Análisis de correlación lineal entre el rendimiento académico y el estilo de aprendizaje del Programa Académico Preparatorio (PAP), abril 2020.

\begin{tabular}{ccccc}
\hline Variable 1 & Variable 2 & $\mathbf{n}$ & $\begin{array}{c}\text { Coeficiente de } \\
\text { correlación de Pearson }\end{array}$ & p-valor \\
\hline Rendimiento académico & Estilo auditivo & 78 & -0.03 & 0.8523 \\
Rendimiento académico & Estilo kinestésico & 39 & -0.16 & 0.5657 \\
Rendimiento académico & Estilo auditivo & \multirow{2}{*}{301} & -0.01 & 0.8892 \\
\hline
\end{tabular}

Fuente: elaboración propia, resultado de investigación.

Según los resultados obtenidos en la tabla anterior ninguno de los estilos de aprendizaje se encuentra relacionado de ninguna manera con el rendimiento académico (notas de 0 a 100 puntos), los coeficientes de correlación de Pearson obtenidos del análisis son muy cercanos a 0 lo cual indica ausencia de relación, los valores probabilidad ( $p$-valor) de los análisis rectifican la falta de relación entre las variables, ya que estos valores están muy por encima del nivel de significancia $(\alpha=0.05)$.

Esta información nos nuestra el principal hallazgo en cuanto a que los estilos de aprendizaje son individuales por lo tanto cada persona es libre de escoger o poseer un estilo de aprendizaje que le permite capturar y guardar a nivel cognitivo la información que percibe a través de los sentidos, y que este estilo no tiene ninguna influencia en el rendimiento académico. 
Para confirmar el hallazgo del presente artículo se indagó en otros estudios como el de Cipagauta (Cipaguata Blanco, 2017) en su tesis titulada “ Los Estilos de Aprendizaje y su relación con del Rendimiento Académico de los Estudiantes de Básica, Secundaria y Media de la Institución Educativa San Agustín”, la investigación se realizó con el propósito de determinar la importancia que tiene conocer los diferentes estilos de aprendizaje en la educación y como estos se relacionan con el rendimiento académico de los estudiantes. El análisis estadístico se efectuó a través de medidas de tendencia central como distribución de frecuencias, media, aritmética, desviación estándar y varianza, determinando que el estilo de aprendizaje con mayor representación es el reflexivo. Luego para obtener la relación entre las dos variables de estilos de aprendizaje y rendimiento académico se utilizó el coeficiente de correlación de $\mathrm{R}$ de Pearson, mediante el cual se identificó que la prueba estadística no identifica relación significativa entre los estilos de aprendizaje y el rendimiento académico de los estudiantes.

Sin embargo, la investigadora hace la recomendación de la importancia de conocer los estilos de aprendizaje para promover la capacitación docente en cuanto al tema.

En el trabajo de tesis de (Velasquez Diaz, 2005) con el tema" Estilos de Aprendizaje en Cálculo I de la Facultad de Ingeniería", de la Universidad Rafael Landívar, el investigador se propuso como objetivo establecer la incorporación de los Estilos de Aprendizaje en los procesos pedagógicos, La investigación de diseño descriptivo, no experimental, realizo un análisis entre los resultados cuantitativos y cualitativos para establecer las recomendaciones pertinentes, la muestra fue constituida por estudiantes y docentes del segundo ciclo de ambos sexos del curso de Cálculo I y autoridades relacionadas con el curso en mención de la Facultad de Ingeniería de la Universidad Rafael Landívar, para un total de 97 personas a quienes se les aplico un cuestionario semicerrado para estudiantes y uno para docentes y autoridades administrativas

De la investigación realizada se obtuvo la opinión generalizada que los estilos de aprendizaje son una herramienta importante para el estudiante y para el docente en la construcción y 
facilitación del conocimiento, resaltando la importancia de la presente investigación en cuanto a indagar acerca de los estilos de aprendizaje de los estudiantes del Programa Académico Preparatorio de la Facultad de Ingeniería. En cuanto a que los estilos de aprendizaje no influyen como concluimos en el rendimiento académico siempre es importante considerar que la mayoría de estudiantes presentan un estilo de aprendizaje visual, lo que le permite al docente mejor su didáctica en cuanto a las necesidades de los estudiantes basado en los resultados de la presente investigación.

\section{Agradecimientos}

EL presente artículo se desarrolló gracias a la colaboración coordinada de la Universidad de San Carlos de Guatemala, la Facultad de Ingeniería y la Coordinación del Programa Académico Preparatorio quienes proporcionaron el apoyo para su desarrollo sobre todo en los tiempos del aislamiento social derivado de la pandemia del Coronavirus (COVID.19)

\section{Referencias Bibliograficas}

Alonzo, C.,. (22 de 03 de 2007). Academia Educativa Los Estilos de Aprendizaje Procedimientos de Diagnóstico y Mejora. España: Ediciones Mensajero. Obtenido de Academia Educativa Los Estilos de Aprendizaje Procedimientos de Diagnóstico y Mejora: Recuperado de https://www. academia.edu/28631359/Alonso_Catalina_M._et.al._Los_ Estilos_de_Aprendizaje

Cipaguata Blanco, Y. L. (2017). Los Estilos de Aprendizaje y su Relación con el rendimiento Académico de los Estudiantes de Básica Secundaria y Media. (Tesis de Maestría). Universidad de Norbert Wiener, Lima Perú.

Figueroa, C. (2009). Sistema de Evaluación Académica. El Salvador: Editorial Universitaria. 
Grinder, J., \& y Bandler, R. (2004). La estructura de la Magia

II. Chile: Recuperado de https://www.academia.

edu/14317545/Estructura_de_la_magia_II_-_Richard_

Bandler_y_John_Grinder. Obtenido de Academia

Educativa: https://www.academia.edu/14317545/

Estructura_de_la_magia_II_-_Richard_Bandler_y_John_

Grinder

Reid, J. (1995). Learming Stlee. U.S.A.: Heile \& Henle Publishers.

Velásquez Diaz, J. A. (2005). Estilos de Aprendizaje en Calculo

I de la Facultad de Ingeniería. (Tesis de Maestria).

Universidad Rafael Landivar, Guatemala, Ciudad.

Woolfolk, A. (2010). Psicología Educativa. México D.F: Pearson

Educacion.

\section{Sobre la autora}

Ingrid Marisol Yumán Ramírez es Maestra en Docencia Universitaria y Licenciada en Psicología de la Escuela de Psicología de la Universidad de San Carlos de Guatemala, con experiencia de siete años en docencia de la USAC y experiencia en Psicología Educativa y en la Administración Educativa, he realizado investigación relacionadas con la administración educativa en cuanto a los manuales de organización y de normas y procedimientos del Sistema de Ubicación y Nivelación de la USAC.

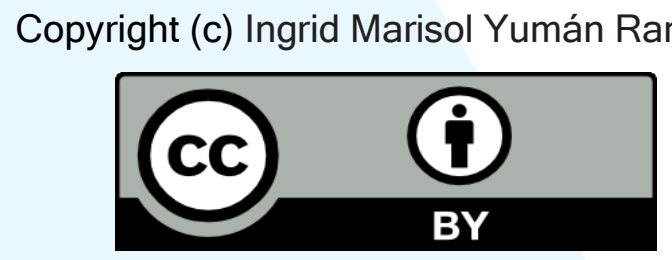

Este texto está protegido por una licencia CreativeCommons 4.0.

Usted es libre para compartir, copiar y redistribuir el material en cualquier medio o formato y adaptar el documento, remezclar, transformar y crear a partir del material para cualquier propósito, incluso comercialmente, siempre que cumpla la condición de atribución: usted debe reconocer el crédito de una obra de manera adecuada, proporcionar un enlace a la licencia, e indicar si se han realizado cambios. Puede hacerlo en cualquier forma razonable, pero no de forma tal que sugiera que tiene el apoyo del licenciante o lo recibe por el uso que hace. 